\title{
Innovative multi-site photoplethysmography measurement and analysis demonstrating increased arterial stiffness in paediatric heart transplant recipients
}

\author{
*Emma J Sharkey ${ }^{1,2}$, *Costanzo Di Maria ${ }^{1,3}$, Annette Klinge ${ }^{1,2}$, \\ Alan Murray ${ }^{3}$, Dingchang Zheng ${ }^{3}$, John O'Sullivan ${ }^{2}$, and John Allen ${ }^{1,3}$ \\ ${ }^{1}$ Microvascular Diagnostics, Northern Medical Physics and Clinical Engineering and \\ ${ }^{2}$ Department of Paediatric Cardiology, Freeman Hospital, Newcastle upon Tyne, NE7 \\ 7DN. ${ }^{3}$ Institute of Cellular Medicine, Faculty of Medical Sciences, Newcastle \\ University. Newcastle upon Tyne, NE1 4LP, UK
}

*Sharkey and Di Maria to be assigned as joint first authors.

Corresponding Author: Dr. John Allen: john.allen@nuth.nhs.uk

Journal:

Physiological Measurement. Special Issue with focus on the analysis and measurement of the arterial pulse wave waveform

Revision: revised version, date: 8/5/18

Manuscript type: Paper ( 3000 words; excluding tables, figures and references).

Short title: pulse wave analysis in paediatric cardiac transplantation

Keywords: arterial stiffness, heart transplant, photoplethysmography, pulse transit time, pulse wave measurement and analysis 


\section{Abstract}

Objective: It has been documented that heart transplantation in children is often complicated by arterial hypertension and increased arterial stiffness. Aim: to use innovative multi-site photoplethysmography (MPPG) pulse measurement and analysis technology to assess arterial stiffness change in paediatric heart transplant recipients $(\mathrm{HTR})$ in comparison with healthy control $(\mathrm{HC})$ children.

Approach: 20 heart transplant (median age 13.5 years old) (8 male) recipients were compared to an overall age- and gender-matched group of 161 healthy controls (11.6 years old) (74 male). The peripheral pulse was recorded bilaterally using MPPG at the ear lobe, index finger and great toe sites, along with an ECG cardiac timing reference. Segmental pulse arrival times between peripheral/sites (finger-ear, PATfe; toe-finger, PATt-f; toe-ear, PATt-e) were calculated as arterial stiffness measures, and differences between subject groups tested using multivariate analysis. Normalised ear, finger and toe pulse shapes were also studied and compared between groups.

Main results: After correction for heart rate, and diastolic and mean blood pressures the HTR group was found to have significantly lower segmental PATt-e and PATt-f measures, with median values of 150 ms versus $172 \mathrm{~ms}$ in $\mathrm{HC}(\mathrm{p}=0.02)$ and $104 \mathrm{~ms}$ (versus $118 \mathrm{~ms}$ in $\mathrm{HC}, \mathrm{p}=0.01$ ), respectively, and consistent with increased arterial stiffness in the patient group. The normalised ear, finger and toe sites showed only a mild elongation in each rise time for the transplant group.

Significance: This study shows innovative and easy-to-do multi-site photoplethysmography gives further evidence for increased arterial stiffness in children who have undergone successful cardiac transplantation. 


\section{Introduction}

Heart transplantation is a main therapeutic option for children and adolescents suffering from cardiomyopathy or end-stage congenital heart disease. This procedure has been shown to bring significant improvement in life expectancy [1-2]. However, health and survival after transplantation is compromised by several factors, including rejection, infection, arterial hypertension, coronary artery disease, graft failure, and malignancies. In particular, arterial hypertension is very common in paediatric heart transplant recipients (HTR) with a prevalence of up to $46 \%$ at one year posttransplantation [3]. The blood pressure elevation in these children has been characterised by systolic blood pressure (SBP) that is mainly elevated at night and diastolic blood pressure (DBP) elevated throughout the 24 hours [4].

The blood pressure elevation noted in this population is likely to be multi-factorial. Increased arterial stiffness is associated with increased cardiovascular risk factor in adults but specific therapies are lacking [5]. Arterial stiffness [6-7] has been often assessed by the pulse wave velocity (PWV) measurement performed using the SphygmoCor mechanical pulse technoblogy [8-11]. A study from our group confirmed that PWV measured using the SphygmoCor device was increased in children posttransplantation when compared to healthy controls [12].

The SphygmoCor system, however, has a number of important disadvantages when used in a clinical setting: it is relatively expensive; and requires a skilled operator to undertake tonometer pulse measurements. Photoplethysmography (PPG) [13] provides an alternative method for assessment of the pulse using simple optical sensor technology. It is relatively low-cost and is quick to perform as pulses from multiple body sites can easily be acquired simultaneously. Our group has already 
demonstrated various clinical applications using multi-site photoplethysmography (MPPG) pulse measurement technology and published data utilising pulse arrival timing measures from different peripheral body sites to assess cardiovascular ageing in normal adult populations [14-17], a pilot in heart transplant patients [18], peripheral arterial disease [19], chronic fatigue syndrome, [20] and cardiovascular function in systemic sclerosis [21]. The aim of this study was to assess arterial stiffness change in paediatric HTR in comparison with healthy children using MPPG technology.

\section{Methods}

\section{Participants}

The study was approved by the County Durham and Darlington Local Research Ethics Committee (05/Q1002/73) and all participants provided written informed consent to participate from their parent or guardian.

Paediatric transplant recipients were recruited from the heart transplant clinic at the Freeman Hospital, Newcastle upon Tyne, UK. Patients were excluded from the study if they were less than 6 years of age, were less than 6 months post-transplantation, had a pacemaker in situ, or had cardiac arrhythmias resulting in unreliable pulse wave analysis. Healthy control $(\mathrm{HC})$ subjects were recruited into the study from local schools. Children who were less than 6 years of age, on beta-blocker or amphetamine medication, a body mass index greater than $30 \mathrm{~kg} / \mathrm{m}^{2}$, who smoked cigarettes, or had had caffeine less than 3 hours before the start of their measurements, were excluded. 
Demographic and other standard clinical variables were collected on the day of measurement: age, height and arm and body segment lengths, weight, body mass index (BMI), and heart rate (HR). Blood pressure was measured manually at the arm using the standard Korotkoff sound method to give the SBP and DBP, from which the mean arterial pressure (MAP i.e. DBP + ((SBP-DBP)/3)) and the pulse pressure (PP i.e. SBP-DBP) were determined.

\section{Pulse wave measurements}

HTR patients were studied in a temperature-controlled room in the hospital with a mean ambient temperature of $25^{\circ} \mathrm{C}$. Healthy controls were studied in a room within the school where the mean study ambient temperature was $25^{\circ} \mathrm{C}$.

MPPG pulses were measured from both the HTR and healthy control participants at the right and left ear lobes, index finger pads and great toe pad sites whilst the subject lay comfortably supine on a measurement couch. The PPG probes (Artema 75333-5 for finger and toe sites, and 75331-9 for ear site) were near infrared (950 $\mathrm{nm}$, with daylight filter) and measured pulses in the reflection mode. A single lead electrocardiogram (ECG) was simultaneously acquired to provide a cardiac timing reference for determining PATs. The physiological waveforms were captured for a period of 2.5 minutes to computer (at $2.5 \mathrm{kHz}, 16$ bit ADC resolution) by one of two operators (EJS and AK). PPG amplifier bandwidth was 0.15 to $20 \mathrm{~Hz}$.

\section{Pulse wave analysis}

The MPPG pulse waveforms were analysed off-line using Matlab-based signal processing and visualization software (The Mathworks Inc.) to provide beat-to-beat 
PAT from the R-wave of the ECG to the foot of each of the 6 PPG pulse waveforms from left and right ear lobe, index finger and great toe. The pulse waveforms were digitally low pass filtered $(20 \mathrm{~Hz})$ and the ECG low pass filtered $(30 \mathrm{~Hz})$ using zero phase filters before the beat-to-beat detection stage for ECG R wave / pulse foot landmark identification for each of the 6 body sites A stage of manual quality checking then facilitated the interpolation of the occasional noisy pulse, for example in the case of pulse shape distortion related to intermittent subject movement artefact [19]. ECG and/or MPPG pulse measurements from any participant that were of very poor quality throughout the recording were excluded from the study.

Timing analysis: The ear pulse transit time (PATe), finger pulse transit time (PATf) and toe pulse transit time (PATt) were each calculated as the median value from 60 consecutive heartbeats, separately for the right and left body sides. It is emphasized that the individual site transit times to the finger or the toe will include the pre-ejection period (PEP), and to eliminate potential differences due to varying PEP, the transit time difference between finger and ear (PATf-e), toe and ear (PATt-e), and toe and finger (PATt-f) were calculated to assess arterial stiffness indirectly, as noted in other studies [22-23] (figure 1).

Shape analysis: The normalized pulse shape at each measurement site was calculated to allow us to qualitatively compare overall characteristics between the HC with HTR participants. The normalized pulse shape profiles were calculated using our previously published methods [24] by using cubic spline interpolation to normalize both the pulse height ( 0 to 1 ) and width (100 pulse width intervals) from 60 consecutive good quality pulses, each overlaid, and an average (median) normalized shape profile determined for each site. 
Statistical analysis

Statistical analysis was performed using Minitab (version 17) and also/SPSS Statistics (version 24, IBM) software. Data were summarised using median, lower (Q1) and upper (Q3) quartiles, written using the form: median (Q1 - Q3). Gender distribution differences between groups were tested using the Fisher's exact test. Paired data differences, e.g. bilateral differences in PAT measure, were tested using the non-parametric Wilcoxon signed rank test. Differences in the clinical characteristics between the controls and the HTR patients were tested by utilising the non-parametric Mann-Whitney $U$ test. Statistical differences in arterial stiffness measures between the healthy controls and the HTR patients were tested using a) univariate analysis utilising the Mann-Whitney $U$ test, and then b) multivariate analysis utilising binary logistic regression (BLR) which corrected for any significant differences in the main clinical characteristics between groups. A p-value less than 0.05 was considered to represent statistical śignificance.

\section{Results}

Twenty eight HTR patients were recruited and 20 of these were included in the analysis. The reasons for exclusion were: presence of a pacemaker (2), too early after transplant (1), incomplete data records or poor signal quality (5). Two hundred and fourteen healthy children were originally recruited and 161 were included in the analysis. The reasons for exclusion were: beta-blocker or amphetamine medication (2), $\mathrm{BMl}>30 \mathrm{~kg} / \mathrm{m}^{2}$ (3), caffeine within 3 hours of measurements (5), smoke cigarettes 
(4), less than 6 years of age when measured (2), incomplete data records (3), and/or very poor ECG / MPPG signal quality for at least one of the measurement sites (40 of which 25 included the ear measurement site).

\section{Clinical variables}

Demographic and clinical characteristics are summarised for the two groups in Table 1. The HTR and the control groups were not statistically different for age and gender distribution. There were no significant differences between groups for height, and body and arm segment lengths or ratios. All children in the HTR group received cyclosprorin. The HTR group had significantly higher HR $(p<0.001)$, DBP $(p<0.01)$, and MAP $(p<0.05)$ and therefore, these three parameters were subsequently adjusted for by using the BLR multivariate analysis.

Segmental body site pulse arrival times

The Wilcoxon signed rank test did not show any significant differences between the right and left side at any of the 3 (ear, finger, toe) segmental levels in healthy controls; hence, measurements from the two sides were averaged together. There was a small but significant difference between right - left ears of HTR patients of $+5.3 \mathrm{~ms}(\mathrm{p}<0.05)$. Summary statistics of pulse arrival time for the two groups are given in Table 2. There were significant differences in site PAT between subject groups for the toes but not the ears or fingers. The segmental difference values for PATt-e, PATt-f were significantly lower in the HTR group, with median (lower quartile - upper quartile) values of 150 (131-161) ms, versus 172 (156-188) ms for controls $(p=0.02)$ and 104 (89-111) ms, versus 118 (109-126) ms for healthy controls, $(p=0.01)$, respectively (figure 2$)$. These differences remained statistically significant 
after correcting for $\mathrm{HR}, \mathrm{DBP}$, and MAP (each at $\mathrm{p}<0.05$ ). No significant difference was found for the PATf-e measure between groups.

\section{Normalised pulse shapes}

There were no overall differences in the normalised pulse shapes between the right and left ears, fingers or toes, and by combining the bilateral sides they were similar to the expected shape characteristics seen in adult subjects [24]. When normalised pulse shapes from $\mathrm{HC}$ and $\mathrm{HTR}$ groups were compared the rising edge appeared to be mildly elongated at each site for the transplant patient group (figure 3 ).

\section{Discussion and Summary}

The increased HR and DBP found in the HTR group are consistent with previous results in the literature $[4,12,25-26]$. Cardiac transplantation results in complete denervation depriving the heart of one of its major rate-regulating mechanisms. In normal subjects, resting HR is predominantly determined by vagal influences so that following cardiac denervation resting HR is higher than normal [25]. The cause(s) of the elevated DBP is not known but is likely to be multifactorial with calcineurin inhibitors and renal dysfunction recognised as important factors in this population. Results on blood pressure parameters from this study and also from Klinge et al. (2009) are consistent with what has been found by O'Sullivan et al. (2005) who showed increased DBP in paediatric HTR over the 24-hour period whereas SBP was elevated only at night. No significant difference in SBP was found in this study. 
Other research groups have shown the feasibility of deriving PATs and other timing and shape measures from PPG [27-29]; however, its potential clinical use requires further investigation, mainly to clarify the effect of possible confounders [17, 30]. Foo et al. (2005) reported a mean toe-finger PAT of $100 \mathrm{~ms}$ in a group of 24 Caucasian children aged 5 to 12 years. This compares to a median value of $118 \mathrm{~ms}$ found in our study. Apart from the fact of using different statistics (mean versus median value), the healthy group assessed in the present study also included children older than 12 years and this could explain the larger value as these subjects would be expected to be taller.

Our study with MPPG has used segmental PAT differences (PATt-e and PATt-f) to overcome the confounding effect of the pre-ejection period. Segmental differences in PAT were clearly found between the healthy control and HTR subject groups and these differences were also statistically significant after adjustment for other clinical variables, i.e. DBP, MAP and HR. It is also interesting to note that this study showed significant differences to the normal population for the PAT to the toe site but not to the finger or ear sites. This may suggest that the arterial rigidity may be increased, at least in children, predominantly in the larger vessels (descending aorta) than the medium sized vessels such as the carotid and brachial arteries. This clearly requires further study. Furthermore, we have shown that the overall normalised pulse shapes in HTR are not grossly different to normal and with only apparent mild elongation in the rise time of the normalised shape pulses at the ear, finger and toe sites for the patient group. We have reported the triangulation and subtle elongation of pulse characteristics with age [24] and our further work will include a detailed comparison of pulse timing and shape characteristics between our adult cohort and this paediatric cohort to further explore aging and PPG waveforms. 
Although it is not essential to measure the segmental limb lengths to get measurements of arterial stiffness, the MPPG technique, using segmental site timing comparisons, can be used to estimate the pulse wave velocity (PWV) if the appropriate lengths are measured [31]. In this study the PPG derived PWV was determined as a feasibility study in a subset of 11 of the HTR patients where reliable body segment length measurements were available and allowing comparisons with the healthy control group. As may have been expected, the PWV from the subset was significantly higher in the HTR group $(6.0 \mathrm{~m} / \mathrm{s}$ versus $5.3 \mathrm{~m} / \mathrm{s}$ for controls; $p<0.001$, unpublished data). This is in keeping with the study by Klinge et al. (2009) where the adjusted aortic PWV was $5.3 \mathrm{~m} / \mathrm{s}$ for HTR patients and $4.7 \mathrm{~m} / \mathrm{s}$ for healthy children obtained using the SphygmoCor device. PWV data in children are relatively uncommon in the literature and the data available is further complicated by the fact that different anatomical sites and different technologies have been used. Some authors used PWV values from proximal sites such as differences between ear and finger [15] and others PWV from finger and toe site differences [30]. Foo et al. studied in an Asian population a group of 55 children, 39 males and 16 females, aged 5 to 12 years old, and reported a mean PWV of $7.3 \mathrm{~m} / \mathrm{s}$.

The causes of the abnormal blood pressure in children following heart transplantation requires further study. Increased rigidity of the arterial system in elderly patients is characterised by higher SBP and lower DBP, hence a larger pulse pressure [32-33]. This is not the pattern observed in children following heart transplant and it is likely therefore that increased rigidity is not the complete explanation. A combination of factors including increased rigidity, denervation, renal dysfunction, and calcineurin inhibitors must be considered in investigating pathophysiology. The optimal drug therapy is, likewise, not known and it is possible that the therapeutic effects on arterial stiffness, as well as blood pressure, should be routinely measured when assessing response to drug therapy. Furthermore, Dall et al. (2015) [34] and 
Passcualino et al. (2015) [35] both showed that a programme of exercise therapy did not reduce the arterial stiffness in HTR. The clinical acceptability and feasibility of our multi-site photoplethysmography technique means that this could be considered as' part of sequential assessment of the vascular system in heart transplantation cardiovascular research. We also believe that our unique pulse data sets from both healthy children and those having had a heart transplant will contain valuable information regarding autonomic function and endothelial function - these aspects are being explored by the team and to be reported on [36-37].

Limitations of the study: We recognise that, although unlikely, the delays seen in HTR might result from the actual surgery and subsequent possible scarring of tissue, there was however no evidence of pulse damping overall for the HTR group; although we did not measure the sympathetic tone at the legs as this could be a source of vasoconstriction and pulse delay, however although the PPG "DC" low frequency changes for PPG at the toes did not reflect such activity in this study we will explore further the signal variability aspect for the two subject groups and we aim to report this in a subsequent publication.

In summary, this study has shown that significantly increased arterial stiffness in children who have successfully undergone cardiac transplantation has been measured using our simple multi-site photoplethysmography technology, demonstrating that the technology can provide relatively rapid and reliable assessment of arterial stiffness changes in this population. The assessment of arterial stiffness in these patients would be beneficial to target appropriate blood pressure management and inform the choice of specific medication and therapy for individual patients. 


\section{Acknowledgments}

The authors thank Dr Richard Kirk (Consultant Paediatric Cardiologist) and Sister Julie Flett (Cardiac Transplant Sister) for supporting us in the study of the transplant patients. We also wish to thank students and teachers at the following schools and colleges for their support: a) Burnside Business and Enterprise College (Wallsend), b) Norham Community College, Collingwood Primary School (North Shields), and also c) Rockcliffe First School and Marden Bridge Middle School (Whitley Bay), North Tyneside, UK.

\section{Funding source}

Supported by a grant from the Special Trustees of Freeman Hospital, Newcastle upon Tyne (Funding reference RES/0146/7216).

\section{Relevant disclosure}

Between 2014 and 2018 Dr John Allen was the Chief Investigator on an NIHR i4i funded grant (II-C1-0412-20003) to develop a miniaturized version of the MPPG technology and specifically for peripheral arterial disease (PAD) detection in a primary care setting. He is a co-author on one published patent and also on one filed patent in relation to the GP MPPG device i.e. the pulse analysis algorithm for PAD detection and also a novel pulse sensor and attachment clip, respectively. 


\section{References}

1. K. Minami, E. von Knyphausen, T. Niino, et al. Long-term results of paediatric heart transplantation. Ann. Thorac. Cardiovasc. Surg. 11, 386-90 (2005).

2. Y.S. Tjang, H. Stenlund, G. Tenderich, L. Hornik, A. Bairaktaris, R. Körfer. Risk factor analysis in paediatric heart transplantation. J. Heart Lung Transplant. 27, 40815 (2008).

3. M. Boucek, L. Edwards, B. Keck, E. Trulock, D. Taylor, M. Hertz. Registry for the International Society for Heart and Lung Transplantation. J. Heart Lung Transplant. 23, 933-47 (2004).

4. J. O'Sullivan, G. Derrick, J. Gray. Blood pressure after cardiac transplantation in childhood. J. Heart Lung Transplant. 24, 891-5 (2005).

5. S. Najjar, A. Scuteri, E.G. Lakatta. Arterial aging. Is it an immutable cardiovascular risk factor? Hypertens. 46, 454-62 (2005).

6. P. Segers, M.F. O'Rourke, K. Parker, N. Westerhof, A. Hughes, On behalf of Participants of the 2016 Workshop on Arterial Hemodynamics: Past, Present and Future. Towards a consensus on the understanding and analysis of the pulse waveform: Results from the 2016 Workshop on Arterial Hemodynamics: Past, present and future. Artery Res. 18, 75-80 (2017).

7. R.R. Townsend. Arterial Stiffness: Recommendations and Standardization. Pulse (Basel). 4(Suppl 1), 3-7 (2017). 
8. I. Wilkinson, S. Fuchs, I. Jansen, et al. Reproducibility of pulse wave velocity and augmentation index measured by pulse wave analysis. J. Hypertens. 16, 2079-84 (1998).

9. B. Pannier, A. Avolio, A. Hoeks, G. Mancia, K. Takazawa. Methods and devices for measuring arterial compliance in humans. Am. J. Hypertens. 15, 743-53 (2002).

10. S. Millasseau, S. Patel, S. Redwood, J. Ritter, P. Chowienczyk. Pressure wave reflection assessed from the peripheral pulse. Hypertens. 41, 1016-20 (2003).

11. S. Millasseau, A. Stewart, S. Patel, S. Redwood, P. Chowienczyk. Evaluation of the carotid-femoral pulse wave velocity. Hypertens. 45, 222-6, (2005).

12. A. Klinge, J. Allen, A. Murray, J. O'Sullivan. Increased pulse wave velocity and blood pressure in children who have undergone cardiac transplantation. J. Heart Lung Transplant. 28, 21-5 (2009).

13. J. Allen. Photoplethysmography and its application in clinical physiological measurement. Physiol. Meas. 28, R1-R39 (2007).

14. C. Di Maria, E. Sharkey, A. Klinge, et al. Feasibility of monitoring vascular ageing by multi-site photoplethysmography. Comput. Cardiol. 39, 817-20 (2012).

15. D. Zheng, A. Murray. Peripheral arterial volume distensibility: significant differences with age and blood pressure measured using an applied external pressure. Physiol. Meas. 32, 499-512 (2011). 
16. J. Allen, A. Murray. Similarity in bilateral photoplethysmographic peripheral pulse wave characteristics at the ears, thumbs and toes. Physiol. Meas. 21, 369-77 (2000).

17. J. Allen, A. Murray. Age-related changes in peripheral pulse timing characteristics at the ears, fingers and toes. J. Hum. Hypertens. 16, 711-7 (2002).

18. C. Di Maria, E. Sharkey, A. Klinge, et al. A pilot study of photoplethysmographic peripheral pulse transit times in paediatric heart transplant recipients and healthy children. Comput. Cardiol. 40, 491-4 (2013).

19. J. Allen, K. Overbeck, A.F. Nath, A. Murray, G. Stansby. A prospective comparison of bilateral photoplethysmography vs. the ankle-brachial pressure index for detecting and quantifying lower limb peripheral arterial disease. J. Vasc. Surg. 47, 794-802 (2008).

20. J. Allen, A. Murray, C. Di Maria, J.L. Newton. Chronic fatigue syndrome and impaired peripheral pulse characteristics on orthostasis - a new potential diagnostic biomarker. Physiol. Meas. 33, 231-41 (2012).

21. N. McKay, B. Griffiths, C. Di Maria, S. Hedley, A. Murray, J. Allen. Novel photoplethysmography cardiovascular assessments in patients with Raynaud's phenomenon and systemic sclerosis: a pilot study. Rheumatology (Oxford) 53, 185563 (2014).

22. M. Nitzan, B. Khanokh, Y. Slovik. The difference in pulse transit time to the toe and finger measured by photoplethysmography. Physiol. Meas. 23, 85-93 (2002). 
23. M. Okada, S. Kimura, M. Okada. Estimation of arterial pulse wave velocities in the frequency domain: method and clinical considerations. Med. Biol. Eng. Comput. 24, 255-60 (1986).

24. J. Allen, A. Murray. Age-related changes in peripheral pulse shape characteristics at various body sites. Physiol Meas 24, 297-307 (2003).

25. W. Beck, C.N. Barnard, V. Schrire. Heart rate after cardiac transplantation. Circulation 40, 437-45 (1969).

26. A. Radaelli, F. Valle, C. Falcone, et al. Determinants of heart rate variability in heart transplanted subjects during physical exercise. Euro. Heart J. 17, 462-71 (1996).

27. J.Y.A. Foo, C.S. Lim. Pulse transit time as an indirect marker for variations in cardiovascular related reactivity. Technol. Health Care 14, 97-108 (2006).

28. C.F. Clarenbach, A.C. Stoewhas, A.J. van Gestel , et al. Comparison of photoplethysmographic and arterial tonometry-derived indices of arterial stiffness. Hypertens. Res. 35(2), 228-33 (2012).

29. E. von Wowern, G. Östling, P.M. Nilsson, P. Olofsson. Digital photoplethysmography for assessment of arterial stiffness: Repeatability and comparison with applanation tonometry. PLoS One 10(8), e0135659 (2015).

30. F.Y.A. Foo, S.J. Wilson, G. Williams, M-A. Harris, D. Cooper. Age-related factors that confound peripheral pulse timing characteristics in Caucasian children. J. Human Hypertens. 19, 463-6 (2005). 
31. S. Laurent, J. Cockcroft, L. Van Bortel, et al. Expert consensus document on arterial stiffness: methodological issues and clinical applications. Euro. Heart J. 27, 2588-2605 (2006).

32. M. O’Rourke, G. Mancia. Arterial stiffness. J. Hypertens. 17, 1-4 (1999).

33. S. Franklin. Arterial stiffness and hypertension. Hypertens. 45, 349-51 (2005).

34. C.H. Dall, F. Gustafsson, S.B. Christensen, F. Dela, H. Langberg, E. Prescott. Effect of moderate- versus high-intensity exercise on vascular function, biomarkers and quality of life in heart transplant recipients: A randomized, crossover trial. J. Heart Lung Transplant. 34(8), 1033-41 (2015).

35. L.N. Pascoalino, E.G. Ciolac, A.C. Tavares, et al. Exercise training improves ambulatory blood pressure but not arterial stiffness in heart transplant recipients. $J$. Heart Lung Transplant. 34(5), 693-700 (2015).

36. I. Mizeva, C. Di Maria, P. Frick, S. Podtaev, J. Allen. Quantifying the correlation between photoplethysmography and laser Doppler flowmetry microvascular lowfrequency oscillations. J. Biomed. Opt. 20(3), 037007. doi: 10.1117/1.JBO.20.3.037007 (2015).

37. J. Allen, C. Di Maria, I. Mizeva, S. Podtaev. Finger microvascular responses to deep inspiratory gasp using wavelet analysis. Physiol Meas 34, 769-79 (2013) 


\section{Legends}

Figure 1. Example of individual body site pulse transit time (PATe, PATf, and PATt) and their segmental timing difference computation (PATf-e, finger site - ear site; PATt-f, toe - finger; PATt-e, toe - ear). PAT values are calculated using the median of 60 consecutive pulses.

Figure 2. Significantly reduced segmental pulse arrival times in the HTR group for toe-ear (PATt-e) and toe-finger site (PATt-f) segmental timing differences (each $\mathrm{p}<0.005$ without BLR correction; $\mathrm{p}<0.05$ with BLR correction for heart rate, diastolic and mean arterial blood pressures). No significant difference was found for the PATfe measure between groups. The boxplots represent the median (with symbol), IQR and also the $10-90$ percentile values.

Figure 3. Normalised shape profiles for $\mathrm{HC}$ and HTR ear, finger and toe sites. Diagrammatically, there was an overall tendency for mild elongation of the pulse rise time for each site with the transplant patients - as illustrated by the clear early peaks in each HTR-HC difference plot.

Table 1. Summary of the clinical characteristics for the two participant groups. The $p-$ value for the gender difference is from the Fisher's exact test; in all other cases the Mann-Whitney U-test was used. NS indicates not statistically significant.

Table 2. Multi-site photoplethysmography pulse arrival times for the ear lobe, index finger and great toe sites, and their segmental timing differences. The segmental pulse timing differences between finger and toe and between ear and toe sites were statistically significant between the participant groups. The p-values were from the Mann-Whitney U-test. NS indicates not-significant statistically. 
Table 1.

\begin{tabular}{|c|c|c|c|}
\hline & $\begin{array}{l}\text { Healthy Controls } \\
\text { (HC) } \\
\text { Median (Q1 - Q3) }\end{array}$ & $\begin{array}{l}\text { Heart Transplant } \\
\text { Recipients (HTR) } \\
\text { Median (Q1 - Q3) }\end{array}$ & $\begin{array}{c}\text { HC vs HTR } \\
\text { (p-value) }\end{array}$ \\
\hline Subjects (males) & $161(74)$ & $20(8)$ & NS \\
\hline $\begin{array}{c}\text { Age } \\
\text { (years) }\end{array}$ & $11.6(9.2-13.3)$ & $13.5(8.5-16.5)$ & NS \\
\hline $\begin{array}{l}\text { Height } \\
\text { (m) }\end{array}$ & $1.45(1.35-1.58)$ & $1.49(1.26-1.60)$ & NS \\
\hline $\begin{array}{l}\text { Weight } \\
(\mathrm{kg})\end{array}$ & $40.0(29.0-51.5)$ & $40.0(25.2-54.5)$ & NS \\
\hline $\begin{array}{c}\mathrm{BMI} \\
\left(\mathrm{kg} / \mathrm{m}^{2}\right)\end{array}$ & $18.3(15.5-20.5)$ & $16.7(15.2-21.0)$ & NS \\
\hline $\begin{array}{c}\text { Heart Rate } \\
\text { (beats per minute) }\end{array}$ & $78(72-83)$ & $(79-99)$ & $<0.001$ \\
\hline $\begin{array}{c}\text { SBP } \\
(m m H g)\end{array}$ & $105(98-116)$ & $109(101-120)$ & NS \\
\hline $\begin{array}{c}\mathrm{DBP} \\
(\mathrm{mmHg})\end{array}$ & $62(54$ & $73(59-80)$ & $<0.01$ \\
\hline $\begin{array}{c}\text { MAP } \\
(m m H g)\end{array}$ & $77(70-85)$ & $87(70-93)$ & $<0.05$ \\
\hline $\begin{array}{c}\mathrm{PP} \\
(\mathrm{mmHg})\end{array}$ & $44(34-51)$ & $38(30-50)$ & NS \\
\hline
\end{tabular}


Table 2.

\begin{tabular}{|c|c|c|c|}
\hline $\begin{array}{l}\text { Pulse timing } \\
\text { measure }\end{array}$ & $\begin{array}{l}\text { Healthy Controls } \\
\text { (HC) } \\
\begin{array}{l}\text { Median (Q1 - Q3) } \\
\text { (ms) }\end{array}\end{array}$ & $\begin{array}{l}\text { Heart Transplant } \\
\text { Recipients (HTR) } \\
\text { Median (Q1 - Q3) } \\
\text { (ms) }\end{array}$ & \\
\hline Ear PAT & $136(129-147)$ & $139(137-153)$ & \\
\hline Finger PAT & $191(175-204)$ & $189(179-201)$ & 6 \\
\hline Toe PAT & $311(290-324)$ & $292(271$ & $=0.006$ \\
\hline PATf-e & $54(44-62)$ & $48(40-56)$ & NS \\
\hline PATt-f & $118(109-126)$ & $104(89-111)$ & $p<0.001$ \\
\hline PATt-e & $172(156-188)$ & $150(131-161)$ & $p<0.005$ \\
\hline
\end{tabular}


Figure 1.

\section{Pulse timing measures}

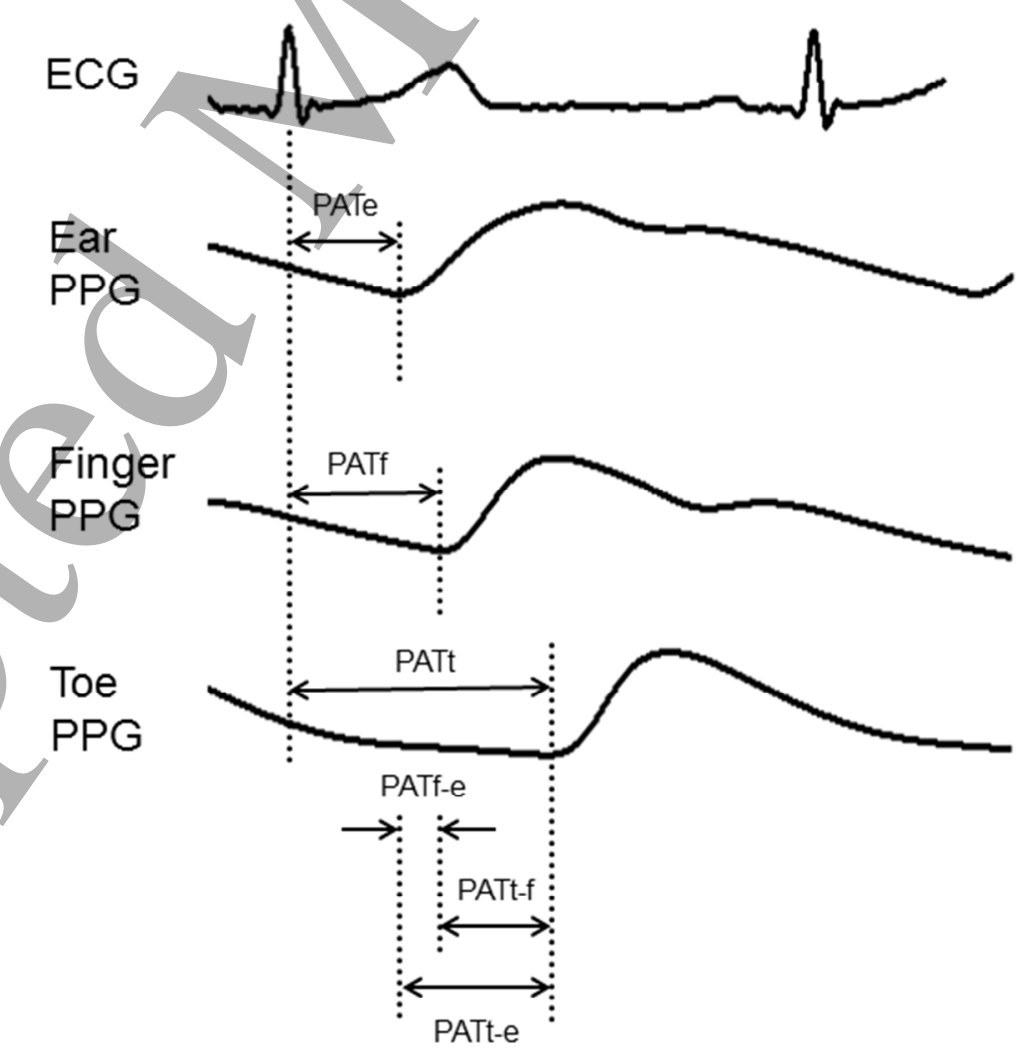


Figure 2.

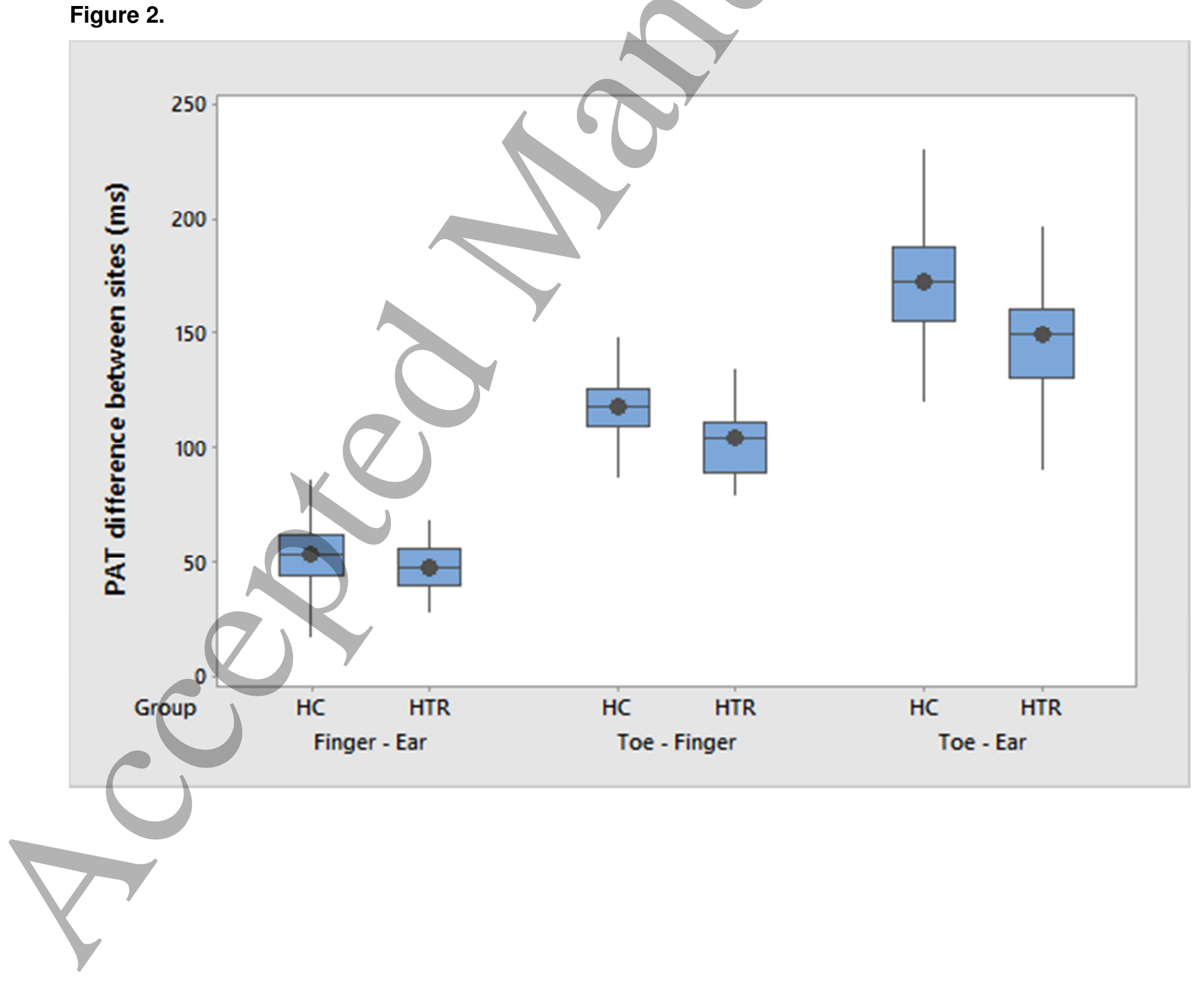


Figure 3.

\section{Normalised pulse shapes: Healthy controls compared to HTR}

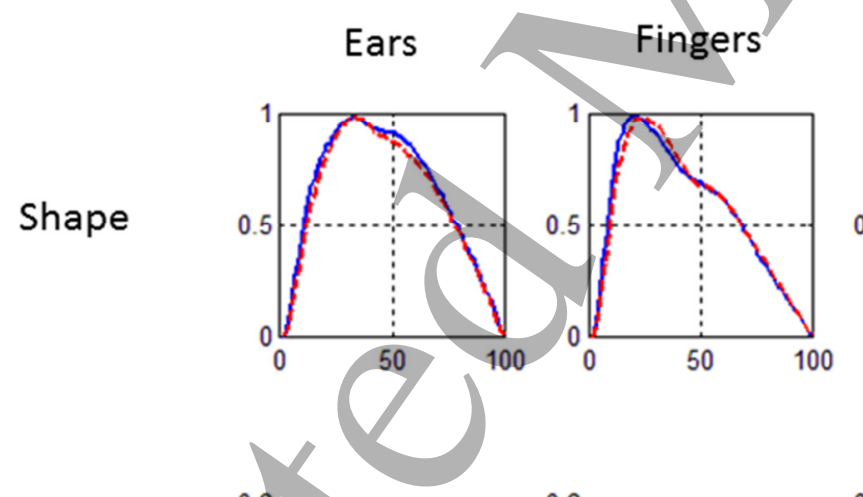

Toes
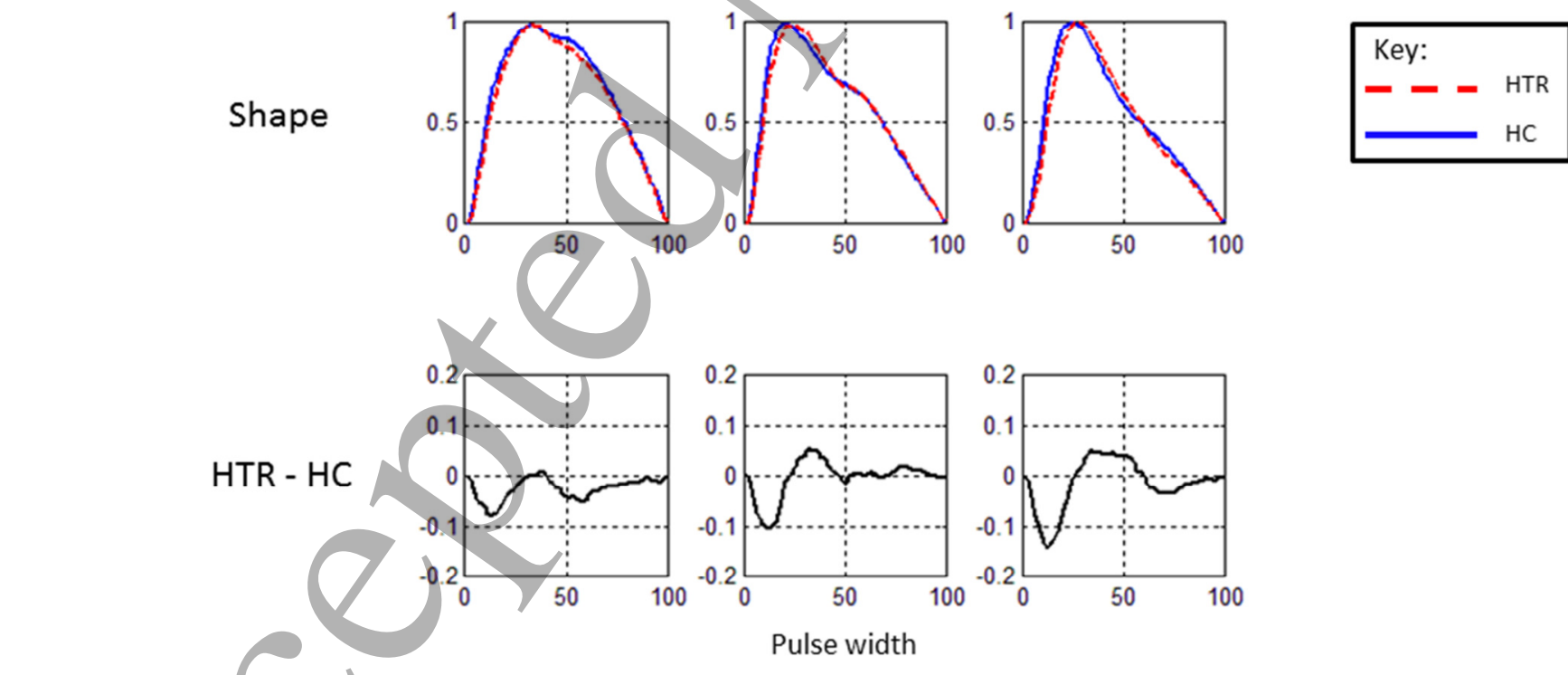